\title{
Development of biogenic silver nano particle from pelargonium graveolens leaf extract and their antibacterial activity
}

\author{
Manonmani Pandian ${ }^{1, *}$, Ramar Marimuthu ${ }^{2}$, Geetha Natesan ${ }^{1}$, Raskin Erusan Rajagopal ${ }^{3}$, \\ Jerlin Sowmiya Justin ${ }^{1}$, Abdul Jaffar Ali Haja mohideen ${ }^{4}$ \\ ${ }^{1}$ Department of Biotechnology, Mother Teresa Women's University, Kodaikanal, India \\ ${ }^{2}$ Department of Zoology, APSA, College,Thiruppattur- 626141,India \\ ${ }^{3}$ Department of Genetics, Dr.A.L.M.Post Graduate Institute of Basic Medical, Sciences, University of Madras, Chennai-600113, India \\ ${ }^{4}$ Department of Biotechnology, Islamiah College, Vaniyambadi - 635752, India

\section{Email address:} \\ pmano_phd@yahoo.co.in (P. Manonmani),ramarer@gmail.com (M. Ramar), geethadrbio@gmail.com (N. Geetha), \\ raskinerusan@gmail.com (R. R. Erusan), sowmiya jelin@yahoo.com(J. J. Sowmiya), jaffar_asc@yahoo.com(H. A. J. Ali)
}

\section{To cite this article:}

Manonmani Pandian, Ramar Marimuthu, Geetha Natesan, Raskin Erusan Rajagopal, Jerlin Sowmiya Justin, Abdul Jaffar Ali Haja mohideen. Development of Biogenic Silver Nano Particle from Pelargonium Graveolens Leaf Extract and their Antibacterial Activity. American Journal of Nanoscience and Nanotechnology. Vol. 1, No. 2, 2013, pp. 57-64. doi: 10.11648/j.nano.20130102.13

\begin{abstract}
Antibacterial activity of biogenic silver nanoparticles was evaluated by zone of inhibition using standard agar disc diffusion method. Among three different concentrations $(25,50$ and $100 \mu 1) 100 \mu 1$ concentration showed maximum activity against Klebsiella pneumonia $(2.7 \mathrm{~cm})$, Shigella someneii $(2.4 \mathrm{~cm})$, S. flexaneri $(2.4 \mathrm{~cm})$, Pseudomonas aeruginosa $(2.2 \mathrm{~cm})$, P. mirabilis $(2.1 \mathrm{~cm})$ and E. coli $(2.0 \mathrm{~cm})$ when compared to the other two concentrations. It was also observed that it showed no activity against geranium plant extract that served as control.
\end{abstract}

Keywords: Pelargonium Graveolens, Geranium Plant Extract, Green Synthesis, Silver Nano Particles, Antimicrobial Activity

\section{Introduction}

Nanotechnology is a field of applied science and can be termed as the synthesis, characterization, exploration and application of nanosized (1-100 nm) materials for the development of science (1, $2 \& 14)$. It deals with the materials whose structures exhibit significantly novel and improved physical, chemical, and biological properties, phenomena, and functionality due to their nano scaled size and have a larger surface area than macro-sized materials. Nanoparticles exhibit a number of special properties relative to bulk material and often have unique visible properties because they are small enough to confine their electrons and produce quantum effects $(3,4 \& 33)$. Nanotechnology is also being utilized in medicine for diagnosis, therapeutic drug delivery and the development of treatments for many diseases and disorders, and also in medical applications on early disease detection, treatment and prevention.

Since noble metal nanoparticles such as gold, silver and platinum nanoparticles are widely applied to human contacting areas, there is a growing need to develop environmentally friendly processes of nano particles synthesis that do not use toxic chemicals (9\& 12). Some physical and chemical methods cannot avoid the use of toxic chemicals in their synthesis $(16,18,17 \& 24)$. The development of consistent processes for the synthesis of silver nano materials is an important aspect of current nanotechnology research. One of such promising process is green synthesis (25). However, for the past few years various rapid chemical methods have been replaced by green synthesis because of avoiding toxicity of the process and increased quality $(10,20 \& 37)$.

Biological methods of nanoparticles synthesis using microorganisms $(8,28 \&$ and 31$)$, plant extracts $(7,5,21$ and $27)$ and enzymes $(22 \& 23)$ have been suggested as possible eco friendly alternatives to chemical and physical methods (34).

Synthesis of nanoparticles using microorganisms, both unicellular and multicellular like yeast, fungi and bacteria have been established, which were able to synthesize 
inorganic materials either extracellularly (32 \& 37) or intracellularly (19). Fungus like Colletotrichum sp., growing on the geranium leaves was used for the synthesis of multiple shaped gold nanoparticles (29), Fusarium oxysporum was used for the synthesis of GNP while Aspergillus fumigatus (6) and A. clavatus (38) used for the synthesis of AgNP within size range of 5 - $25 \mathrm{~nm}$.

Compared to microorganisms which suffer from various problems like availability, maintenance in cell cultures and cost effectiveness during the scale up process, various plants have been successfully used for the synthesis of gold, silver and sometimes for bimetallic silver and gold nanoparticles. A number of plants such as alfalfa (Medicago sativa) produced GNP of 4-10 nm (11). Biomass of oat (Avena sativa) and wheat (Triticum aestivum) were reported for irregular and rod shaped GNP with size range 10-30 nm (3), was also reported for gold nanotriangles. Similarly for the synthesis of AgNP plants such as Capsicum annuum (15), Citrullus colocynthis (27), Coriandrum sativum leaf extract (26) were used. In addition to the independent synthesis of silver and gold nanoparticles various plants such as leaf extract of Aloe vera produced triangular and spherical silver and gold nanoparticles (6), and neem (Azadirachta indica) leaf broth was used for the synthesis of nanoparticles of silver, gold and Ag core-Au shell (29).

Species of Geranium (Pelargonium graveleons) are evergreen perennials indigenous to southern Africa and are drought, heat tolerant and also tolerate only minor frost. An essential oil is extracted from this plant contains diuretic antispasmodic properties. It is known for it's number of medicinal values such as treatment of skin diseases, arthritis, anti inflammatory and also have been reported for it's anticancer activity, antibacterial properties and anti oxidant property (21). The earthy, minty and floral odour of this aromatic oil has been proved extremely effecting in curing burns, tonsillitis, poor circulation, eczema and rheumatism. It also helps in increased circulation and stress related condition.

If biological synthesis of nanoparticles can compete with chemical methods, there is a need to achieve faster synthesis rates. In the present investigation, the synthesis of pure metallic nanoparticles of silver by the reduction of $\mathrm{Ag}+$ using Geranium (Pelargonium graveolens) leaf extract was carried out by adopting rapid technique of synthesis of nanoparticles. The time required for more than $90 \%$ reduction of $\mathrm{Ag}+$ ions using Geranium leaf extract was about $2 \mathrm{~h}$. The exact mechanism of silver nanoparticles synthesis by selected plant extracts is not yet fully understood. Only participation of phenolics, caroboxyl groups, proteins and reducing agents in their synthesis has been speculated ( $29 \& 15)$. In the present study, we have analysed and screened Geranium leaf extract (Pelargonium graveleons) for nanoparticles synthesis and characterized by using UV- visible spectroscopy, XRD, SEM, EDS and FT-IR and also assessed the antimicrobial activity of synthesized silver nanoparticles.

\section{Materials and Methods}

\subsection{Plant Material and Preparation of the Extract}

Fresh Pelargonium graveolens leaves were collected from the Mother Teresa Women's University campus, Kodaikanal (Tamil Nadu) India, is well documented in the Kodaikanal (Horticultural Research Station). The experimental chemicals were purchased from Sigma Chemicals (Mumbai).

\subsection{Synthesis of Silver Nanoparticles}

The fresh Pelargonium graveleons leaves free from diseases were collected, washed thoroughly 2-3 times with tap water and with sterile water, dried at $40 \mathrm{C}$ in hot air oven and powdered and then used for extraction. About 2 grams of dried leaf powder were ground to fine paste with $20 \mathrm{ml}$ of distilled water using mortar and pestle. It was centrifuged at 10,000 RPM for 10 minutes and supernatant was taken for further processing. Ten $\mathrm{ml}$ of the plant leaf extract was added to $90 \mathrm{ml}$ of aqueous solution of $5 \mathrm{mM}$ Silver nitrate solution $\left(\mathrm{AgNO}_{3}\right)$ for reduction of silver nitrate into $\mathrm{Ag}+$ ions and maintained at room temperature $\left(37^{\circ} \mathrm{C}\right)$ in the incubator in static condition and the completion of the reaction was carried out for a period of $2 \mathrm{~h}$. The colourless silver nitrate solution is changed from pale yellow to ruby red and finally dark brown colour which indicates the formation of silver nanoparticles.

\subsection{UV-Visible Spectroscopy Analysis}

The colour change in reaction mixture (Silver nitrate solution + leaf extract) was recorded through visual observation. The bio reduced silver nanoparticle solution was filtered through Whatmann No.1 filter paper and the filtrate was measured using UV-Visible absorbance. The bioreduction of silver ions in aqueous solution was monitored by periodic sampling of aliquots $(1 \mathrm{ml})$ and subsequently measuring UV-vis spectra of the solution using Double beam UV-vis spectrophotometer (model 2201) operated at a resolution of $1 \mathrm{~nm}$.

\subsection{X-Ray Diffraction (XRD) Measurements}

The completely bioreduced sample was concentrated in concentrator (Eppendorf) at $50^{\circ} \mathrm{C}$ to reduce the volume of the reaction mixture. The concentrated solution was then centrifuged at $12000 \mathrm{rpm}$ for 15 minutes. The pellet obtained, was washed and redispersed in deionized water. Repeated centrifugation and redispersion in deionized water was carried out to remove the water soluble biomolecules such as proteins and secondary metabolites. XRD measurements of powdered purified silver nanoparticle thus obtained was performed on a Shimazdu, model Lab X-XRD-6000 instrument operated at a voltage of $40 \mathrm{kV}$ and a current of 30 $\mathrm{mA}$ with $\mathrm{Cu} \mathrm{K} \alpha$ radiation with a wavelength of $1.5406 \AA$. 


\subsection{Determination of Crystalline Size}

Average crystallite size of silver was calculated using the Scherrer's formula,

$\mathrm{D}=\mathrm{k} \lambda / \beta \cos \theta$

$\mathrm{D}=$ Average crystallite size: $\mathrm{K}-$ Constant: $\lambda$ - X-ray Wavelength: $\beta$ - Angular FWHM of the

XRD peak at the diffraction angle: $\theta$ - Diffraction angle.

\subsection{FTIR (Fourier Transform Infrared Spectroscopy) Analysis}

After the complete reduction of $\mathrm{Ag}+$ ions by the geranium leaf extract and the pellets of silver nanoparticles was centrifuged at $12000 \mathrm{rpm}$ for $15 \mathrm{~min}$ and the resulting suspension was re dispersed into $20 \mathrm{ml}$ of distilled water, the process of centrifuging and re dispersing was repeated three times to make nano particles free from proteins or other bioorganic compounds present in the solution. There after the purified suspension was completely dried in lyophilizer and analyzed by PerkinElmer-Spectrum RX-IFTIR.

\subsection{SEM \& EDS Analysis of Silver Nanoparticles}

The pellet was subjected for SEM analysis. Thin films of the sample were prepared on a carbon coated copper grid by just dropping a very small amount of the sample on the grid, extra solution was removed using a blotting paper and then the film on the SEM grid were allowed to dry for analysis. A scanning electron microscope (JEOL 6380A; Tokyo, Japan) was used to record the micrograph images of synthesized Ag-NPs. Energy-dispersive x-ray (EDS) spectroscopy analysis for the confirmation of elemental silver was carried out for the detection of elemental silver.

\subsection{Antibacterial Assay}

Antibacterial activity of the silver nanoparticles was assessed using the standard agar disc diffusion method with $6 \mathrm{~mm}$ diameter Whatmann No.1 filter paper discs (29 \& 30). In this method $25 \mu l, 50 \mu l$, and $100 \mu l$ of silver nano particles prepared from synthesized form leaf extract mixed in one $\mathrm{ml}$ of ethanol and applied to sterile paper discs of 6 $\mathrm{mm}$ diameter and plant extract without nano particles was used for control. Bacteria such as P. aeruginosa, P. mirabilis, E. coli, Shigella flexaneri, S. somenei and Klebsiella pneumonia was used for the antimicrobial test. Before the antibacterial assay a log-phase culture of bacteria such as $P$. aeruginosa, P. mirabilis, E. coli, Shigella flexaneri, $S$. somenei and Klebsiella pneumonia were inoculated into the nutrient agar plates and incubated at $37^{\circ} \mathrm{C}$ for 24 hours. Zone of Inhibition was measured after $24 \mathrm{~h}$ of incubation.

\section{Results and Discussion}

\subsection{UV-Visible Spectra Analysis}

The synthesized Ag nano particles using geranium plant extracts confirmed by visual observation. The colour was changed from pale yellow to ruby red due to reduction of silver ions during the reaction. The appearance of dark yellowish-brown colour is a clear indication of the formation of silver nano particles in the reaction mixture (fig.1). Ag nanoparticle were detected by UV-Vis spectrophotometer in range of 350 to $620 \mathrm{~nm}$. The maximum absorption spectra of silver nano particles formed in the reaction mixture has increasingly sharp absorbance maximum peak at $440 \mathrm{~nm}$. It is well known that $\mathrm{Ag}$ nano particles exhibits reddish brown colour in aqueous solution due to excitation of surface plasmon vibrations. There is a spectral shift is mainly due to the dielectric constant of the medium (5). The colour exhibited by metallic nanoparticles is due to the coherent excitation of all the "free" electrons within the conduction band, leading to an in-phase oscillation which is known as Surface Plasmon Resonance-SPR (4). Here silver nanoparticles synthesized using geranium plant extract were stable for more than one month, when stored at room temperature. The frequency and width of the surface plasmon absorption depends on the size and shape of the metal nanoparticles as well as on the dielectric constant of the metal itself and the surrounding medium ( $22 \& 23)$. In the present study the reduction of $\mathrm{Ag}+$ ions occurs rapidly, completing within $2 \mathrm{~h}$ of reaction. The silver nanoparticles obtained from the reaction mixture containing $5 \mathrm{mM} \mathrm{AgNO}_{3}$ and $2 \mathrm{~g}$ of dried leaf powder were purified and further analyzed.

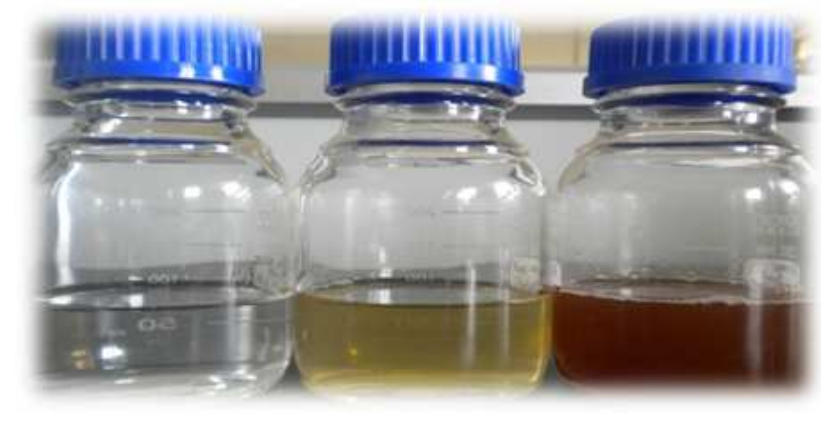

Fig 1. Photographs of (a) $\mathrm{AgNO}_{3}$ (b) Leaf extract (c) Leaf extract $+\mathrm{AgNO}_{3}$ after $2 h$ of reaction

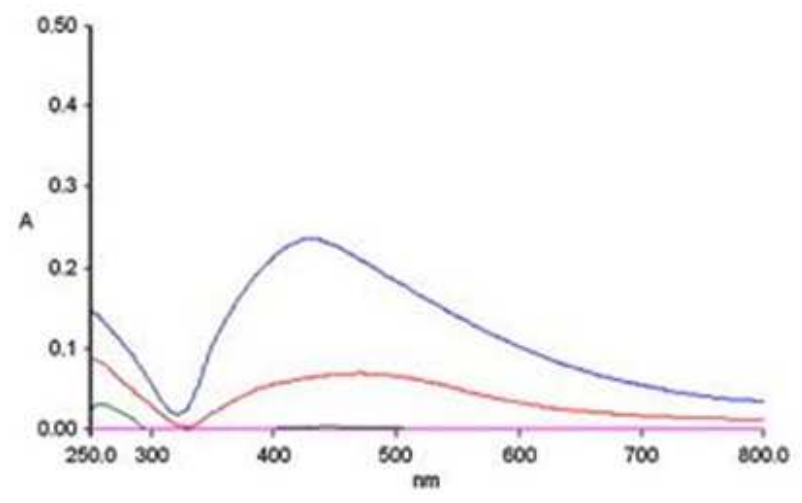

Fig 2. UV-vis spectra of reduction of Ag ions to Ag nano particles

(27) reported rapid synthesis of stable silver, gold and bi-metallic $\mathrm{Ag} / \mathrm{Au}$ core shell nanoparticles using $20 \mathrm{~g}$ of leaf biomass of Azadiracta indica and $1 \mathrm{mM}$ aqueous $\mathrm{AgNO}_{3}$. 
The present study reports the use of oven dried leaf biomass, which is free from external stabilizing and accelerating agents and does not require continuous agitation. The reduction of the silver ions is moderately rapid at the ambient conditions. This is novel and intriguing to the material science as the studied leaf biomass has the capability to reduce metal ions at ambient conditions.
Furthermore the biomass handling and processing is less stringent since it does not require boiling or subsequent treatment. Instead of sun drying the leaf biomass was oven dried at $40^{\circ}$ to avoid the possible contamination due to particulate matter. Also the sun drying process is time consuming and has geographical constraints.

Table 1. Crystalline size of silver nanoparticles synthesized using geranium leaf extract

\begin{tabular}{|c|c|c|c|c|c|}
\hline Plant extract & $\begin{array}{c}\theta \text { value } \\
\text { (degree) }\end{array}$ & d- spacing (̊̊) & FWHM (degree) & Intensity (CPS) & $\begin{array}{l}\text { Average } \\
\text { Particle size } \\
\text { (nm) }\end{array}$ \\
\hline Geranium Silver nanoparticles & 16.125 & 0.2667 & 2.77291 & 100.00 & 47.29 \\
\hline
\end{tabular}

The XRD pattern showed nine intense peaks in the whole spectrum of $2 \theta$ values ranging from 27.8 to 77.2 in synthesied nanoparticle of geranium. XRD pattern obtained for silver nanoparticles showed a characteristic peaks near the $2 \theta$ value of $38.145^{\circ}$ (Fig. $3 \mathrm{~b}$ ). The peaks observed in the spectrum at $2 \theta$ values of $38.145^{\circ}, 44.31^{\circ}, 46.58^{\circ}$ and $64.44^{\circ}$ corresponds to $111,200,220$, and 311 planes for silver, respectively. This clearly indicates that the silver nanoparticles formed by the reduction of $\mathrm{Ag}+$ ions by the Pelargonium graveolens leaf extract are crystalline in nature
(13). A Bragg reflection corresponding to the (111) sets of lattice planes are observed which may be indexed based on the face - centered cubic (fcc) structure of silver (27). In addition to the Bragg peak representative of fcc silver nanocrystals, additional and yet unassigned peaks were also observed suggesting that the crystallization of bio-organic phase occurs on the surface of the silver nanoparticles.

The average estimated particle sizes of the samples were calculated using the Debye-Scherrer formula. An average size of the particles synthesized by geranium was $47 \mathrm{~nm}$.

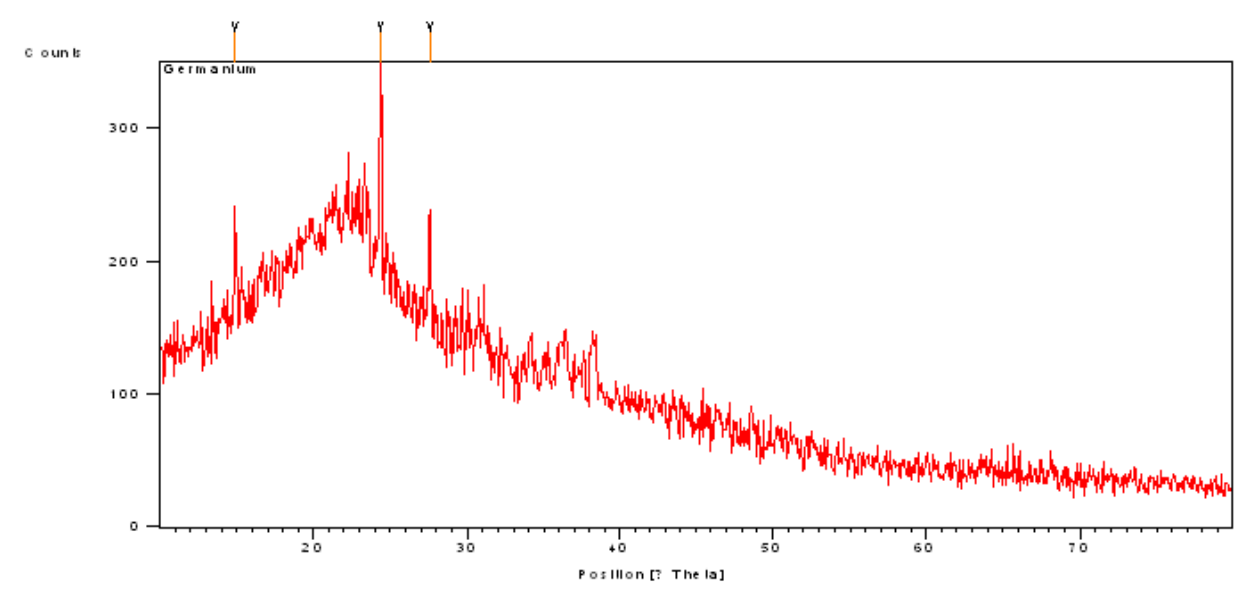

Fig 3a. XRD patterns of Geranium (Pelargonium graveolens) dried leaf powder

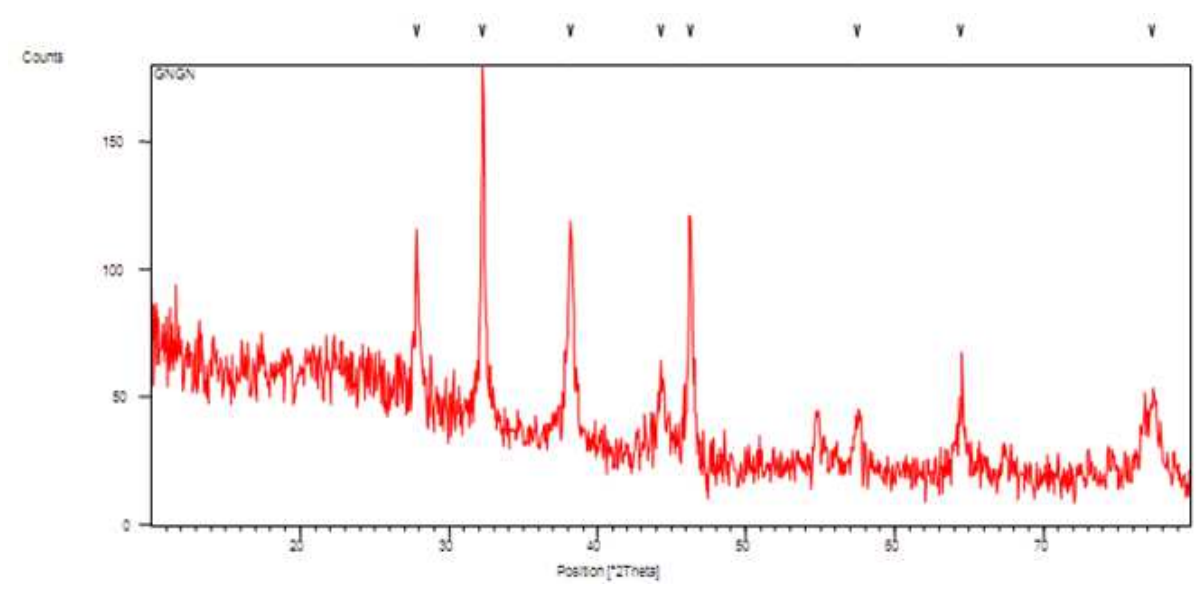

Fig 3b. XRD patterns of silver nanoparticles synthesized using dried leaf powder of Pelargonium graveolens. 
The scanning electron microscopic (SEM) image showed high density Ag nanoparticles synthesized by the plant extract and further confirmed the presence of $\mathrm{Ag}$ nanoparticles (Figs.4a and 3b). SEM image has showed individual silver particles as well as a number of aggregates. The observed morphology of the silver nanoparticles was predominately cuboidal, and rectangular, uniform, poly dispersed and aggregated into larger irregular structure with no well-defined morphology (fig.4a \&b). Ag nanoparticles were formed with diameter of 13 to $61 \mathrm{~nm}$. The SEM image of silver nanoparticles were synthesized from plant extracts are assembled on to the surface due to the interactions such as hydrogen bond and electrostatic interactions between the bio-organic capping molecules bound to the $\mathrm{Ag}$ nanoparticles. The nanoparticles were not in direct contact even within the aggregates, indicating stabilization of the nanoparticles by a capping agent (proteins secreted by plant leaf extracts). The presence of secondary materials capping with the silver nanoparticles may be assigned to bio-organic compounds from leaf extracts agent (33).
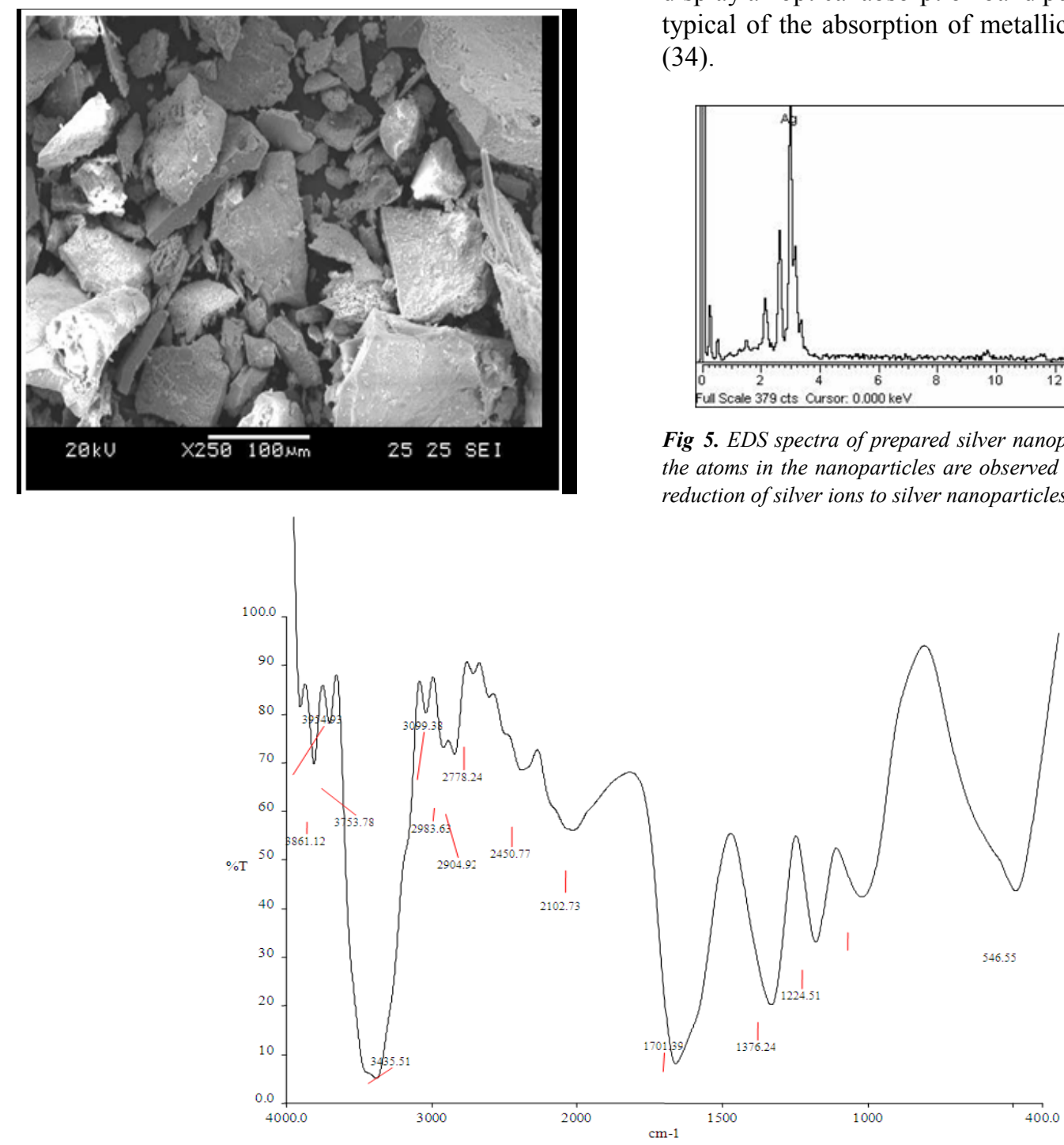

Fig 6. Showing the FTIR spectrum of synthesised silver nano particles of geranium

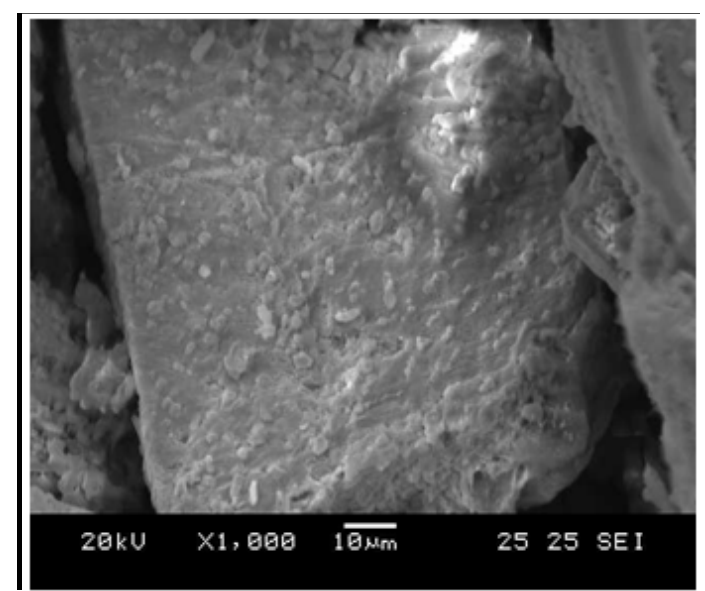

Fig 4. SEM images of Ag Nanoparticles (a) 250000 magnification (b) 1 Lakh magnification.

The results of Energy dispersion Spectroscopy (EDS) confirms the presence of elemental silver signal in the sample and is shown in fig.5. The $\mathrm{Ag}$ nanocrystallites display an optical absorption band peaking at $3 \mathrm{keV}$ which is typical of the absorption of metallic silver nanocrystallites (34).

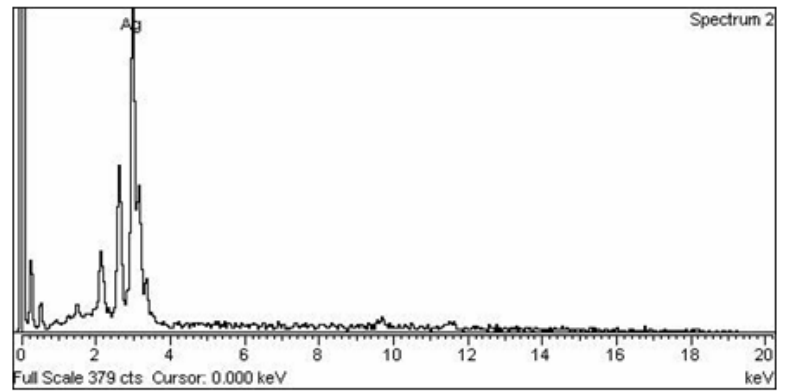

Fig 5. EDS spectra of prepared silver nanoparticles. Strong signals from the atoms in the nanoparticles are observed in spectrum and confirm the reduction of silver ions to silver nanoparticles 
In FTIR spectrum the wave number or frequency (cm-1) of absorption band or peak assigned to the type of vibration, intensity and functional groups of the silver nanoparticles. FTIR measurements were performed to identify the potential biomolecules in the geranium leaf extract (Pelargonium graveolens) responsible for reduction and then providing stability to the bioreduced silver nanoparticles. In the FTIR spectrum several absorption peaks were centered at 3954.93, 3861.12, 3753.78, 3435.51, 3099.38, 2983.63, 2904.92, $2778.24,2450.77,2102.73,1701.39,1376.24,1224.51$ and $1069.55 \mathrm{~cm}-1$ which was in the region range of $1000-4000$ cm-1 (figure6). The most wide spectrum absorption was observed at $3435.51 \mathrm{~cm}-1$ and it can be attributed to the stretching vibrations of -NH (amide groups). The absorption peaks centered at 2904.92, 2102.73, 1701.39 and1376 cm-1 can be attributed to the stretching vibrations of $-\mathrm{C}=\mathrm{C}$ (alkane) groups, $-\mathrm{C}$ - $\mathrm{D}$ deuterated alkanes groups, $\mathrm{C}=\mathrm{O}$ simple $\square$ lactams, amide I band groups and $\mathrm{C}-\mathrm{H}$ (-CH3 band) of alkanes groups. Now various functional groups mentioned above are mainly derived from heterocyclic compounds and these are the water soluble components of geranium leaf extract. So it can be assumed that different water soluble heterocyclic compounds such as alkaloids, flavonoids etc., worked as the capping ligand for the synthesis of silver nanoparticles and the presence of oxygen atoms helped in the stabilization of nanoparticles by facilitating the absorption of heterocyclic compounds on nanoparticles. FT-IR analysis reveals that the carbonyl group from amino acid residues and proteins has the stronger ability to bind metal indicating that the proteins could possibly form a layer covering the metal nanoparticles (i.e., capping of silver nanoparticles) to prevent agglomeration and thereby stabilize the medium. This suggests that the biological molecules could possibly perform dual functions of formation and stabilization of silver nanoparticles in the aqueous medium.

\subsection{Antimicrobial Studies}

Antibacterial activity of biogenic silver nanoparticles was evaluated by zone of inhibition using standard agar disc diffusion method. The nano particles showed inhibition zone against all the studied bacteria (Fig.7). Among three different concentrations $(25,50$ and $100 \mu l) 100 \mu l$ concentration showed maximum activity against Klebsiella pneumonia $(2.7 \mathrm{~cm})$, Shigella someneii $(2.4 \mathrm{~cm})$, S. flexaneri $(2.4 \mathrm{~cm})$, Pseudomonas aeruginosa $(2.2 \mathrm{~cm})$, P. mirabilis $(2.1 \mathrm{~cm})$ and E. coli $(2.0 \mathrm{~cm})$ when compared to the other two concentrations. It was also observed that it showed no activity against geranium plant extract that served as control. (36) investigated the antibacterial effect of nanosized silver colloidal solution against $S$. aureus and $K$. pneumoniae after padding the solution on textile fabrics. The antimicrobial properties of silver compounds and silver ions had been historically recognized and applied in a wide range of applications from disinfecting medical devices and home appliances for the treatment of water. The silver nano particles produced by plant extracts are known to exhibit potent antimicrobial activity. A similar observation has been made with the silver nano particles produced from geranium plant extract.

The antibacterial activity is probably derived, through the electrostatic attraction between negative charged cell membrane of microorganism and positive charged nanoparticles ( $7 \& 8)$. However, (36 \& 35) reported that the antimicrobial activity of silver nanoparticles on Gram-negative bacteria was dependent on the concentration of $\mathrm{Ag}$ nanoparticles and was closely associated with the formation of pits in the cell wall of bacteria.

(35) studied antibacterial activity against E. coli (ampicillin resistant), E. coli, S. aureus, and S. typhi (multi-drug resistant). They reported that the effect was dose dependent and was more pronounced against gram-negative organisms than gram-positive ones. They found that the major mechanism through which silver nanoparticles manifest antibacterial properties was either by anchoring or penetrating the bacterial cell wall and modulating cellular signaling by dephosphorylating putative key peptide substrates on tyrosine residues (36).

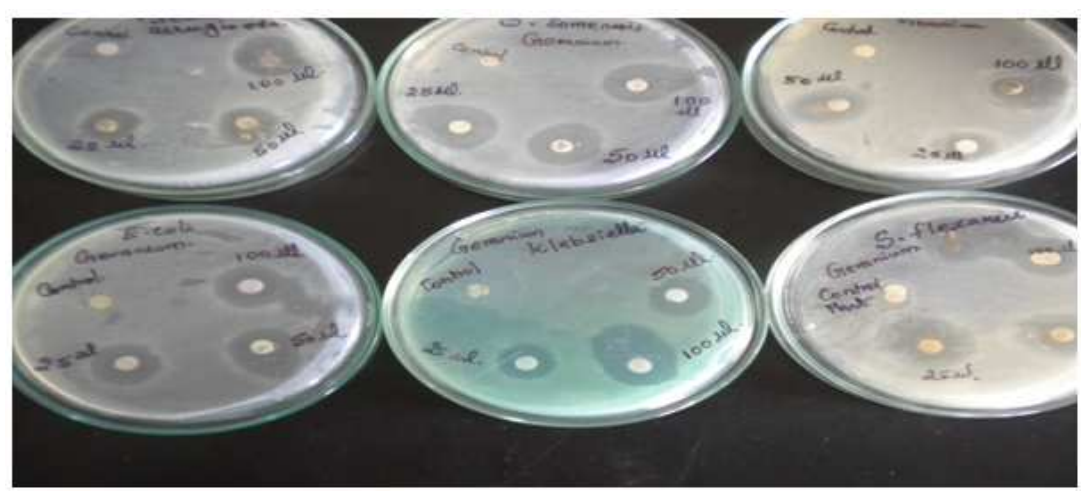

Fig 7. Antibacterial activity of synthesised nano particles from geranium leaf extract 


\section{Conclusion}

In this study we present a simple, low cost, clean, nontoxic and rapid approach for the bio reduction and synthesis of silver nano particles. This method provides an efficient route for the synthesis of nano particles with tunable optical properties directed by particle size. From the point of view of nanotechnology this is a significant advancement to synthesize silver nano particles economically. The silver nano particles show distinct poly dispersity as it shows particle size between the ranges of 13 to $61 \mathrm{~nm}$ with an average size of $49 \mathrm{~nm}$. The identification of coumpounds, capping agents and tapping the enzymes responsible for the reduction process may open up new avenues in the field of nanotechnology and nanoparticle stabilization. Investigation on the antibacterial effect of nanoparticles against $P$. aeruginosa, P. mirabilis, E. coli, Shigella flexaneri, S. somenesis and Klebsiella pneumonia reveals high efficacy of silver nanoparticles as a strong antibacterial agent. This can be useful in food industries, cosmetic industries and medicines.

\section{Acknowledgements}

The authors acknowledge the Mother Teresa Women's University for providing necessary facilities. We extend our heartfelt gratitude to DST-CURIE for funding to carry out this project successfully.

\section{References}

[1] Ahmad, A., Senapati, S., Khan, M. I., Kumar, R. and M. Sastry. 2003. Extracellular biosynthesis of mono disperse gold nanoparticles by a novel extremophilic actinomycete, Thermomonospora sp. Langmuir, 19: 3550 - 3553.

[2] Ahmad, A., Senapati, S., Khan, M. I, Ramani, R., Srinivas, V. and M. Sastry. 2003 a. Intracellular synthesis of gold nanoparticles by a novel alkalo tolerant actinomycete Rhodococcus species. Nanotechnology. 14: 824 - 828.

[3] Armendariz, V.. Gardea-Torresdey, J. L., Jose-Yacaman, M., Gonzalez, J., Herrera, I. and J. G.Parsons. 2002. In Proceedings -Waste Research Technology Conference at the Kansas City, Mariott-Country Club Plaza.

[4] Bhattacharya, D and R.K. Gupta. 2005. Nanotechnology and potential of microorganisms. Critical Reviews in Biotechnology, 24(4), 199.

[5] Chandan Singh, Vinect Sharma, Pradeep, K. R Naik, Vikas Khandelwal and Harvinder Singh. 2011. A green biogenic approach for synthesis of gold and silver nanoparticles using Zingiber officinale. Digest Journal of nanomaterials and Biostructures. 6 (2): 535-542.

[6] Chandran, S. P., Chauhary, M., Pasricha, R., Ahmad, A. and M. Sastry. 2006. Synthesis of gold nanotriangles and silver nanoparticles using aloe Vera plant extract. Biotechnol. Prog., 22: 577-583
[7] Dibrov, P., Dzioba, J., Gosink, K. K. and C. C. Hase. 2002. Antimicrobial Agents. Chemother., 46: 2668 - 2670.

[8] Dragieva, I., Stoeva, S., Stoimenov, P., E. Pavlikianov and K. Klabunde, 1999. Extracelluar sysnthesis of silver nanoparticles using dried leaves. Nanostruct. Mater., 12: 267 -72 .

[9] Elumalai, E. K., Prasad, T.N.V.K.V., Hemachandran, J., Viviyan Therasa, S., Thirumalai, J and E. David. 2010. Extra cellular synthesis of silver nanoparticles using leaves of Euphorbia hirta and their antibacterial activities. J. Pharm. Sci. and Res., 2(9): 549- 554.

[10] Emad al din Haratifar, Hamid Reza Shahverdi, Mojtaba Shakibaie, Kamyar Mollazadeh Moghaddam, Mohsen Amini, Hojatollah Montazeri and Ahmad Reza Shahverdi. 2009. Semi-biosynthesis of Magnetite-gold composite nano particles using an ethanol extract of Eucalyptus camaldulensis and study of the surface chemistry. Journal of Nanomaterials, 2: 1-5.

[11] Gardea-Torresdey, J. L., Parson, J. G., Gomea, E., Peraltaviden, J. R., Troiani, H. E., Santiago, P. and M. J. Yacaman. 2002. Formation and growth of Au nanoparticles inside live alfalfa plants. Nano Lett., 2: 397- 401.

[12] Gardea- Torresdey, J. L. and E. Gomez. 2003. Alfalfa sprouts: A natural source for the synthesis of silver nanoparticles. Langmuir, 19: 1357 - 1361.

[13] Jegan, A., Ramasubbu, A., Vasanth Kumar, S., Balamurugan, M. and S. Saravanan . 2011 Environmental benign synthesis and characterization of silver nanoparticles using Phyllostachys sp leaves extract. Digest Journal of Nanomaterials and Bio structures. 6 (1): 325-330

[14] Kamat, P.V. 2002. Photophysical, photochemical and photocatalytic aspects of metal nanoparticles. J. Phys. Chem. B., 106:7729-7744.

[15] Li, S., Shen, Y., Xie, A., Yu, X., Qiu, L., Zhang, L. and Q. Zhang. 2007. Green synthesis of silver nanoparticles using Capsicum annuum L. extracts. Green Chem., 9: 852 -858.

[16] Link, S., Mohamed, M.B., EI-Sayed, M. A.1999. Simulation of the optical absorption spectra of gold nanorods as a function of their aspect ratio and the effect of the medium dielectric constant. J. Phys. Chem. B., 103: $3073-3077$.

[17] Ma, H., Yin, B., Wang, S., Jiao, Y., Pan, W., Huang, S., Chem, S. and F. Meng. 2004. Synthesis of silver and gold nanoparticles by a novel electrochemical method. Chem Phys. Chem., 5: 68-75.

[18] Malikova, N,, Pastoriza-Santos, I,, Schierhorn, M., Kotov, N. A. and L. M. Liz-Marzan. 2002. Layer-by-layer assembled mixed spherical and planar gold nanoparticles: control of inter particle interactions. Langmuir, 18: 3694 - 3697.

[19] Mann, S. and G. A. Ozin. 1996. Synthesis of inorganic materials with complex form Nature. 382: 313- 318.

[20] Mano Priya, M., Karunai Selvi, B. and J.A. John Paul. 2011. Green synthesis of silver nanoparticles from the leaf extracts of Euphorbia hirta and Nerium indicum. Digest Journal of Nanomaterials and Bio structures. 6 (2): 869 -877.

[21] Mona Safaepour, Ahmad Reza Shahverdi, Hamid Reza 
Shahverdi, Mohammad Reza khorramizadeh and Ahmad Reza Gohari. 2009. Green synthesis of small silver nanoparticles using geraniol and its cytotoxicity against fibrosarcoma Wehi 164. Avicenna J. Med. Biotech. 1(2): $111-115$.

[22] Mukherjee, P., Ahmad, A., Mandal, M., Senapati, S., Sainkar, S. R., Khan, M. I., Ramani, R., Parischa, R. and P. V. Ajayakumar. 2001. Bioreduction of $\mathrm{AuCl}_{4}$ - ions by the fungus, Verticillium sp. and surface trapping of the gold nanoparticles formed. Angew. Chem.,Int. Ed., 40: 3585 3588 .

[23] Mukherjee, P., Senapati, S., Ahmad, A., Khan, M. I. and M. Sastry. 2002. Extracellular synthesis of gold nanoparticles by the fungus Fusarium oxysporum. Chem. Biochem., 3: 461-463.

[24] Perma, P. Chemical mediated synthesis of silver nanoparticles and its potential antibacterial application. Progress in Mollecular and Environmental Bioengineeringfrom analysis and modelling to technology Applications. 151 $-166$.

[25] Rajesh W. Raut., Niranjan, S., Kolekal., Jaya R. Kakkakula, Vijay D. Mendhulkal and Sahebrao B. Kashid. 2010. Extracellular synthesis of silver nanoparticles using dried leaves of Pongamia pinnata (L) Pierre. Nano-micro Letters, 2(2): $106-113$.

[26] Sathyavathi, R., M. Balamurali Krishna., S. VenugopalRao., R. Saritha and NarayanaRao. 2010. Biosynthesis of Silver nanoparticles using Coriandrum sativum leaf extract and their Application in Nonlinear Optics. Adv. Sci Letters, 3: 1 5.

[27] Satyavani, K., Ramanathan, T and S. Gurudeeban. 2011. Green synthesis of silver nanoparticles by using derived callus extract of bitter apple (Citrullus colocynthis). Digest Journal of Nanomaterials and Biostructures, 6(3): 10191024.

[28] Shah Verdi, Ar., Minaeian, S., Shahverdi, H.R., Jamalifar, H. and A. A. Nohi. 2007. Rapid synthesis of silver nanoparticles using culture supernatants of enterobacteria: a novel biological approach. Process Biochem., 42:919-923.
[29] Shankar, S.S., Rai, A., Ahmad, A. and M. Saatry. 2004. Rapid synthesis of $\mathrm{Au}, \mathrm{Ag}$ and bimetallic $\mathrm{Au}$ core- $\mathrm{Ag}$ shell nanoparticles using neem (Azadirachta indica leaf broth). $J$. Colloid Interface Sci. 275: 496 -502.

[30] Shankar, S. S., Rai, A., Ankamwar, B., Singh, A., Ahmad, A. and M. Sastry. 2004a. Biological synthesis of triangular gold nanoprisms. Nat.Mater, 3: 482 - 488.

[31] Shiying He, Yu Zhang, Zhirui Guo, and Ning Gu. 2008. Biological synthesis of gold nanowires using extract of Rhodopseudomonas capsulate. Biotechnol. Prog., 24: 476-480.

[32] Simkiss K, and K.M. Wilbur. 1989. Biomineralization; Cell biology and mineral deposition. New York: Academic Press; p. 337.

[33] Song, J.Y. and B. S. Kim. 2008. Biological synthesis of bimetallic $\mathrm{Au} / \mathrm{Ag}$ using persimmon (Diopyros kaki) Leaf extract. Korean. J. chem. Eng., 25(4): 808 - 812.

[34] Song, K.C., Lee, S. M., Park, T. S. and B. S. Lee. 2009. Preparation of colloidal silver nanoparticles by chemical reduction method. Korean. J. Chem. Eng., 26(1): 153 -155.

[35] Srivastava, R., Roseti, D., Sharma, A.K.2007. The evaluation of microbial diversity in a vegetable based cropping system under organic farming practices. Applied Soil Ecology, 36(2-3): $116-123$.

[36] Szczepanowicz, K., Stefariska, J., Socha, R. P. and P. Warszyriski. 2010. Preparation of silver nanoparticles via chemical reduction and their antimicrobial activity. Physiochem. Probl. Miner. Process. 45: 85 -98.

[37] Udayasoorian, C., Vinoth Kumar, K. and R. M. Jayabalakrishnan. 2011. Extracellular synthesis of silver nanoparticles using leaf extract of Cassia auriculata. Digest Journal of Nanomaterials and Bio structures. 6 (1): 279-283.

[38] Vijay C Verma, Santosh, Singh, K., Ravidra Solanki and Satya Prakash. 2011. Biofabrication of anisotropic gold nanoangles using extract of endophytic Aspergillus clavatus as a dual functional reductant and stabilizer. Nanoscale Res. Letters. 6(16): 1-7. 